\section{Nutrition in aging and disease: update on biological sciences}

\author{
Holly Brown-Borg*1, Rozalyn Anderson², Roger McCarter ${ }^{3}$, John Morley ${ }^{4}$, \\ Nicolas Musi ${ }^{5}$, S Jay Olshansky ${ }^{6}$, Barbara Shukitt-Hale7, Peggy St. Jacques ${ }^{8}$, \\ Jeremy Walston ${ }^{9} \&$ Bradley Willcox ${ }^{10}$
}

\section{4th Annual Meeting of the Gerontological Society of America 18-22 November 2011, Boston, MA, USA}

The Annual meeting of the Gerontological Society of America brings together four unique sections of the Society to present their latest research findings. With over 4000 in attendance this year, the meeting served as a major opportunity for cross-fertilization and exchange of ideas both within and between sections. This year, the Biological Sciences Section focused their symposia on 'Nutrition in aging and disease'. This translational topic was of great interest and drew robust audiences from each of the societies' sections (the Health Sciences, Behavioral and Social Sciences, as well as the Social Research, Policy and Practice sections). A summary of each of the sessions is presented.

\section{Gerobesity: the coming epidemic}

The global epidemic of obesity has reached into every socioeconomic strata in every nation. The problem is not just adult-onset obesity that began decades ago contributing to the high prevalence of adult obesity; there is a second wave of obesity that has emerged among children that does not bode well for future generations of middle aged and older persons. With medical technology improving to save the lives of people who suffer from the complications of obesity, humanity faces the potential for an explosive increase in the number of people who reach older ages having been obese for decades. In this session, scientists from a range of disciplines discussed the causes of the global obesity epidemic, methods for ameliorating its causes, and the consequences and implications of current and future trends in obesity on trends in period life expectancy in developed nations.

\section{Anorexia of aging: appetite, food intake \\ \& nutrient sensing in the aged}

This session highlighted that anorexia occurs physiologically in both animals and humans with advanced aging. Anorexia has multiple causes such as changes in taste and smell, alterations in stomach fundal compliance, increased activity of the gut satiety hormone, cholecystokinin and alterations in the functioning of central neuropeptides. Leptin, the hormone from adipocytes, also plays a role in this anorexia and interacts with the effects of exercise. Attempts to decrease the loss of muscle mass with aging have led to the development of a myostatin antibody. Unfortunately, the lack of myostatin alters tendons and bones, thus limiting mobility of animals. There is the need for the development of drugs that can enhance appetite and increase muscle strength in older persons.

\section{Insights into genetics \& nutrition in the long-lived}

This session presented evidence for the influence of genetics and nutrition on aging and longevity from observational studies in humans. The principal focus was on caloric intake, markers of energy balance (e.g., BMI), other nutrition-related factors and genetics in several different populations including Ashkenazi Jews, Okinawans and other Japanese in Japan and Hawaii, as well as black and white centenarians and other elderly individuals in the state of Georgia. Epidemiologic evidence for 'dietary restriction', the best-known nutritional intervention affecting healthspan and lifespan in model organisms, was found in the Okinawa Centenarian Study, with an extension of both mean and maximum lifespan. Several micronutrients also appeared important in the Okinawan diet. The Kuakini Hawaii Lifespan Study, a cohort study with over four decades of follow-up carried out in 8006 middle-aged men, found some similar factors as found in the Okinawans, with markers of energy balance, particular micronutrients, fasting glucose, insulin sensitivity and alleles from the $\mathrm{FOXO3}$ energy-sensing gene linked to survival and

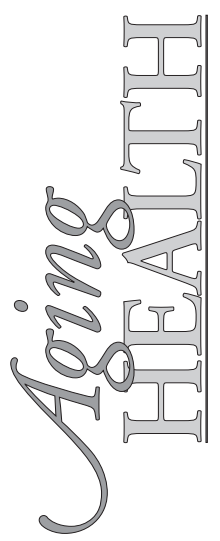

'Department of Pharmacology, Physiology \& Therapeutics, University of North Dakota School of Medicine \& Health Sciences, 501 N Columbia Road, Grand Forks, ND 58202, USA ${ }^{2}$ Department of Medicine \& GRECC, VA Hospital, Madison, WI, USA ${ }^{3}$ College of Health \& Human Development, The Pennsylvania State University, University Park, PA, USA ${ }^{4}$ Saint Louis University School of Medicine, The Department of Internal Medicine, Division of Geriatric Medicine, St Louis, MO, USA

${ }^{5}$ San Antonio GRECC, Audie L Murphy VA Medical Center, San Antonio, TX, USA

${ }^{6}$ School of Public Health, University of Illinois at Chicago, Chicago, IL, USA 'Jean Mayer USDA HNRCA at Tufts University, Boston, MA, USA ${ }^{8}$ Department of Psychology, Harvard University, Cambridge, MA, USA ${ }^{9}$ Bayview: Department of Medicine Geriatrics, Bayview Care Center, Baltimore, MD, USA

${ }^{10}$ Department of Geriatric Medicine, John A Burns School of Medicine, University of Hawaii \& Department of Research, Kuakini Medical Center, Honolulu, HI, USA

*Author for correspondence:

Tel.: +1 7017773949

Fax: +1 7017774490

holly.brown.borg@med.und.edu

\section{Keywords}

- aging biology $\bullet$ genetics

- longevity $\bullet$ nutrition

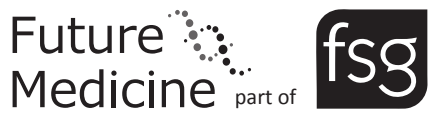


healthy survival to very old age. The Georgia Centenarian Study highlighted the importance of a systems approach to understanding healthy aging, demonstrating how nutrition, lifestyle, personality, social factors and other factors interact to produce healthy aging. This systems approach was embodied by a healthy 105-year-old physician from Japan, who highlighted several keys to healthy aging including diet and physical activity. Overall, while some challenges were seen for obtaining nutritional information from questionnaire-based data, the session provided support for nutritionrelated effects on lifespan and healthspan in humans and suggested a possible role for calorie restriction (CR) and energy-sensing pathways.

\section{5 years of caloric restriction research: where are we now?}

Current knowledge of mechanisms of aging has been greatly expedited by the fact that reduced nutrient input (or CR) extends longevity in a wide variety of animal species. CR is the most reliable environmental manipulation known to slow aging processes, but the underlying mechanisms remain unknown. However, practical applications for benefiting the health of the elderly are obvious, and were addressed by several speakers. CR for 6 months in adult men and women reduced mass-specific metabolic rate more than expected from the associated loss of metabolic mass, while the expected decreases in oxidative damage were not found. Other tissues were also significantly affected. These studies are ongoing and are expected to yield important insights into possible benefits of CR for elderly men and women. Work described in animals points to metabolic reprogramming as an important aspect in the mechanisms of $\mathrm{CR}$. Based on the inverse relationship between calorie intake and lifespan extension, it was shown that regulators of energy metabolism are important. Nutrient-sensitive metabolic regulators may thus be effective targets for treatment of multiple age-associated diseases and disorders. The issue of CR mimetics, agents that might provide health benefits in the absence of reduced food intake, was also addressed. This is an active area of research given the importance of possibly extending human healthspan and reducing morbidity in old age. Compounds such as the plant polyphenol, resveratrol or mannoheptulose (a promising candidate) that mimic CR could prevent or reverse many of the pathological outcomes of a high-fat diet. The beneficial impact of resveratrol treatment on multiple parameters of health in animals subjected to diet-induced obesity was shown. Gene expression in tissues from resveratrol-treated animals identified a role for genes involved in metabolism. The high translatability of this work on CR mimetics is nicely illustrated in a recent study where resveratrol improved health in obese human subjects.

\section{Nutrition in brain aging \& neurodegenerative disease}

The onset of age-related neurodegenerative diseases such as Alzheimer's disease (AD) or Parkinson's disease, superimposed on a declining nervous system, could exacerbate the motor and cognitive behavioral deficits that normally occur in senescence. Thus, the exploration of methods to retard or reverse age-related neuronal deficits is extremely important to increase healthy aging. Consumption of foods and nutrients high in polyphenols in addition to prudent-type diets (i.e., high in fruits, vegetables, whole grains and fish) has been shown to prevent and even reverse the occurrence of neurochemical and behavioral changes that occur in aging. Conversely, consuming a typical western-style diet associates with increased risk of cognitive decline and dementia. Pleiotropic agents, including an omega-3 fatty acid (docosahexanoic acid), the turmeric spice curcumin and exercise can target multiple steps in the $\mathrm{AD}$ cascade including amyloid and tau oligomers, inflammation, oxidative damage and synaptic toxicity. Furthermore, AD prevention approaches may need genetically informed applications. Muscle mass and strength both decline with age, but the loss of strength far surpasses what is projected based on the decline in mass. The accumulation of fat mass has been shown to be a strong independent predictor of subsequent functional loss and disability. Aging and a high-fat diet similarly impaired muscular strength/function as well as molecular signaling associated with muscle growth pathways. These data support the need to promote healthy lifestyles, including healthy eating, beginning in mid-adulthood as a means of supporting cognitive health in an aging population.

\section{Contributions of inflammation \& muscle-related gene variants to frailty \& late-life decline}

The use of human genetics studies to identify biological pathways relevant to longevity has yielded clues that predict good health, but few studies have looked at frailty and the pathways 
that link it to late-life decline and early mortality. Such studies could provide insights into the development of chronic diseases, sarcopenia and generalized late-life decline. The Johns Hopkins Older Americans Independence Center (Baltimore, MD, USA) focuses on the identification of biological etiologies of frailty through phenotype and genetic study development. This symposium presented related findings from the Cardiovascular Health Study, a population of approximately 5600 communitydwelling older adults living in four regions of the USA. The activation of inflammatory pathways predicts both frailty and other adverse health outcomes such as mortality in older adults. Two cytokines were identified (IL- 6 and $s$ TNF- $\alpha$ R1) as the most powerful predictors of mortality over 10 years (a finding validated in a second population study of 1300 older Italians [in CHIANTI]). Genetic data showed that polymorphic variation in genes related to mitochondrial biology, DNA methylation and skeletal muscle function are associated with the same two cytokines. A skeletal muscle endophenotype of grip strength and walking speed (that underlies frailty) was also found to be associated with the same group of gene polymorphisms. These investigators identified a relationship of the candidate genes with the complete human frailty phenotype. This work suggests that common biological pathways related to DNA methylation, skeletal muscle differentiation and maintenance and mitochondrial biogenesis may be important in accelerated late-life decline and frailty. Although clinical implications from these data are not clear at the present time, the inflammatory phenotype data will aid in generalized risk assessment in older patients and in the development of targeted preventive efforts to decrease pathological inflammatory pathway activation.

\section{Functional neuroimaging of autobiographical memory}

Studies investigating the influence of age on the neural mechanisms supporting autobiographical memory, or memory of our personal past, have rapidly increased in the last year. One presentation related evidence suggesting that aging preserves a common memory retrieval network but reduces the selectivity of engaging specific networks supporting autobiographical and episodic memory. Another talk suggested that providing more supportive and personalized retrieval cues, generated using a novel camera technology, can attenuate age-related differences in autobiographical recollection by allowing older adults to recruit compensatory brain mechanisms. One investigator highlighted the overlap between age-related differences in autobiographical memory retrieval and the ability to understand other mental states. The last presentation examined the contribution of autobiographical memory to planning for the future and found that aging affects the ability to modulate neural networks according to task demands. The research showcased in the symposium expands our understanding of age-related changes in memory by examining processes that are difficult to study with laboratory memory, such as the rich recollection of the past and memories linked to other mental states and future prospection, which make our personal memories meaningful and important.

\section{Oxidative stress \& inflammation: role in the pathophysiology of age-related diseases}

Oxidative damage to macromolecules (e.g., DNA, proteins and lipids) is associated with several age-related diseases. Oxidative stress and mitochondrial dysfunction also lead to a proinflammatory state. One presentation described data from a study that utilized a unique model in which the antioxidant enzyme superoxide dismutase was overexpressed in transgenic rats, and demonstrated the impact that this genetic intervention had on lifespan, glucose tolerance, and insulin sensitivity. Other work discussed the roles that oxidative stress and protein aggregation play on the pathogenesis of amyotrophic lateral sclerosis. The third presentation described how impaired aldehyde detoxification contributes to the aging process and Parkinson's disease. Lastly, a new model for the temporal evolution of $\mathrm{AD}$ pathology was proposed based on combined autopsy data with latent growth curve models of cognitive performance. Overall, this session highlighted the importance of oxidative stress and inflammation on the pathogenesis of several age-related diseases and provided evidence that modulating oxidative damage can alter the course of some of these pathologies.

\section{Conclusion}

One of the overarching messages from the multitude of research presented at sessions sponsored by the Biological Sciences Section at the 2011 Gerontological Society of America meeting is that an individual's genetics play a role in longevity and aging processes, but 
maintaining a healthy environment (including dietary intake, social influences and exercise) contribute greatly to healthy aging and slowing age-related disease incidence and progression.

\section{Financial \& competing interests disclosure}

Seven symposia were cosponsored by the National Institute on Aging (NIH AG038099 and AG038099-02S1), the Ellison Medical Foundation (AG-CW-0344-11), the
Glenn Foundation for Medical Research and AGE-UK. The remaining three symposia were submitted. The authors have no other relevant affliations or financial involvement with any organization or entity with a financial interest in or financial conflict with the subject matter or materials discussed in the manuscript apart from those disclosed.

No writing assistance was utilized in the production of this manuscript. 